\title{
RESEARCH
}

Open Access

\section{Cerebrospinal fluid a-synuclein predicts neurodegeneration and clinical progression in non-demented elders}

Jie-Qiong Li ${ }^{1,2,3}$, Yan-Lin Bi ${ }^{4}$, Xue-Ning Shen ${ }^{1}$, Hui-Fu Wang ${ }^{2}$, Wei X ${ }^{2}$, Chen-Chen Tan ${ }^{2}$, Qiang Dong ${ }^{1}$, Yan-Jiang Wang ${ }^{5}$, Lan Tan ${ }^{2^{*}}$, Jin-Tai Yu ${ }^{1 *}$ (D) and Alzheimer's Disease Neuroimaging Initiative

\begin{abstract}
Background: Accumulating reports have suggested that a-synuclein is involved in the pathogenesis of Alzheimer's disease (AD). As the cerebrospinal fluid (CSF) a-synuclein has been suggested as a potential biomarker of AD, this study was set out to test whether CSF a-synuclein is associated with other AD biomarkers and could predict neurodegeneration and clinical progression in non-demented elders.

Methods: The associations between CSF a-synuclein and other AD biomarkers were investigated at baseline in non-demented Chinese elders. The predictive values of CSF a-synuclein for longitudinal neuroimaging change and the conversion risk of non-demented elders were assessed using linear mixed effects models and multivariate Cox proportional hazard models, respectively, in the Alzheimer's disease Neuroimaging Initiative (ADNI) database.

Results: The CSF a-synuclein levels correlated with AD-specific biomarkers, CSF total tau and phosphorylated tau levels, in 651 Chinese Han participants (training set). These positive correlations were replicated in the ADNI database (validation set). Using a longitudinal cohort from ADNI, the CSF a-synuclein concentrations were found to increase with disease severity. The CSF a-synuclein had high diagnostic accuracy for AD based on the "ATN" (amyloid, tau, neurodegeneration) system (A $+\mathrm{T}+$ versus $\mathrm{A}-\mathrm{T}$ - control) (area under the receiver operating characteristic curve, 0.84). Moreover, CSF a-synuclein predicted longitudinal hippocampus atrophy and conversion from $\mathrm{MCl}$ to $\mathrm{AD}$ dementia.

Conclusions: CSF a-synuclein is associated with CSF tau levels and could predict neurodegeneration and clinical progression in non-demented elders. This finding indicates that CSF a-synuclein is a potentially useful early biomarker for AD.
\end{abstract}

Keywords: Alzheimer's disease, a-Synuclein, Biomarker, Cerebrospinal fluid, Neurodegeneration

\footnotetext{
* Correspondence: dr.tanlan@163.com; jintai_yu@fudan.edu.cn

${ }^{2}$ Department of Neurology, Qingdao Municipal Hospital, Qingdao University, Qingdao 266071, China

'Department of Neurology and Institute of Neurology, Huashan Hospital, Shanghai Medical College, Fudan University, Shanghai 20040, China Full list of author information is available at the end of the article
}

C C The Author(s). 2020 Open Access This article is licensed under a Creative Commons Attribution 4.0 International License, which permits use, sharing, adaptation, distribution and reproduction in any medium or format, as long as you give appropriate credit to the original author(s) and the source, provide a link to the Creative Commons licence, and indicate if changes were made. The images or other third party material in this article are included in the article's Creative Commons licence, unless indicated otherwise in a credit line to the material. If material is not included in the article's Creative Commons licence and your intended use is not permitted by statutory regulation or exceeds the permitted use, you will need to obtain permission directly from the copyright holder. To view a copy of this licence, visit http://creativecommons.org/licenses/by/4.0/. The Creative Commons Public Domain Dedication waiver (http://creativecommons.org/publicdomain/zero/1.0/) applies to the data made available in this article, unless otherwise stated in a credit line to the data. 


\section{Background}

Alzheimer's disease (AD) is the leading cause of dementia in the elderly and is clinically characterized by a gradual decline in memory and other cognitive functions. However, less than half of the patients with dementia have received a formal diagnosis in Europe and the USA [1]. The pathological change of $\mathrm{AD}$ can precede the onset of clinical symptoms by 20 years. Biomarker research has made it possible to identify people at the high risk of developing dementia in the general population, even at the preclinical stage $[2,3]$. According to the newly published "ATN" scheme, various biomarkers can be divided into three binary components: (i) biomarkers of $\beta$-amyloid $(\mathrm{A} \beta)$ plaques or associated pathophysiologic processes labeled as "A"; (ii) biomarkers of aggregated pathologic tau or associated pathophysiologic processes labeled as "T"; and (iii) biomarkers of neurodegeneration or neuronal injury labeled as " $N$ " [4]. Besides the biomarkers mentioned above, additional novel biomarkers that reflect other disease mechanisms may provide insights into the different mechanisms of $\mathrm{AD}$ pathogenesis and assist in identifying novel therapeutic targets in the future. This was echoed by the 2018 NIA-AA research framework that "ATN" can be expanded to incorporate other proteinopathies that are also involved in AD pathogenesis or frequently co-occur with $\mathrm{AD}$ pathologic changes [5-7]. This provided a multidimensional approach to diagnosing dementia and for better clinical stratification of patients for therapeutic trials $[8,9]$.

$\alpha$-Synuclein is best known for its roles in Parkinson's disease (PD) and dementia with Lewy bodies (DLB), and has also been reported to be implicated in AD pathogenesis [10]. Patients with $\mathrm{AD}$ and concomitant $\alpha$-synuclein pathology typically have a more rapid rate of cognitive decline than those with AD alone [11, 12]. $\alpha$-Synuclein is generally considered as a pre-synaptic protein, which can also be found in human cerebrospinal fluid (CSF) $[13,14]$. Many studies have reported differences in the CSF $\alpha$-synuclein levels between PD and control [15-17] as well as the diagnostic differentiation of different neurodegenerative diseases $[18,19]$. However, the potential role of CSF $\alpha$-synuclein as a biomarker for the presymptomatic phase of $\mathrm{AD}$ remains unclear.

In this study, we explored the associations between CSF $\alpha$-synuclein and other AD biomarkers in the nondemented Chinese elderly. We also tested whether CSF $\alpha$-synuclein was altered in patients with $A D$ and with different pathophysiological profiles of $\mathrm{AD}$ based on the "ATN" classifications, and its associations with other AD biomarkers, cognitive decline and imaging evidence of neurodegeneration in the Alzheimer's Disease Neuroimaging Initiative (ADNI) database. The value of CSF $\alpha$ synuclein as a predictor of disease progression and neurodegeneration at the presymptomatic stage of $\mathrm{AD}$ was also investigated.

\section{Methods}

\section{Study participants}

Six hundred and fifty-one non-demented participants were from the Chinese Alzheimer's Biomarker and Lifestyle (CABLE) study. The CABLE is a large-cohort study mainly focusing on Alzheimer's risk factors and biomarkers in Chinese elderly adults. The participants in the CABLE study were recruited at Qingdao Municipal Hospital, consisting of cognitively normal $(\mathrm{CN})$ and mild cognitive impairment (MCI) individuals. All participants were Han Chinese in origin and aged 50-90 years. The controls had Mini-Mental State Examination (MMSE) scores of 24 or higher, with lower scores indicating more impairment and higher scores less impairment (range, 0-30), and a Clinical Dementia Rating (CDR) score of 0, where lower scores indicate less impairment and higher scores more impairment (range, 0-3). The patients with MCI had MMSE scores of 24 or higher, an objective memory loss tested by delayed recall of the Wechsler Memory Scale (WMS) logical memory II ( $>1$ SD below the normal mean), a CDR score of 0.5, preserved activities of daily living, and absence of dementia. The exclusion criteria were: (1) central nervous system infection, head trauma, epilepsy, multiple sclerosis or other major neurological disorders; (2) major psychological disorders (e.g., depression); (3) severe systemic diseases (e.g., malignant tumors) that may affect CSF or blood levels of $\mathrm{AD}$ biomarkers including $\mathrm{A} \beta$ and tau; and (4) family history of genetic disease. All participants underwent clinical and neuropsychological assessments, biochemical testing, as well as blood and CSF sample collection. Demographic information, AD risk factor profile and medical history were also collected by a comprehensive questionnaire and an electronic medical record system.

Data were obtained from the ADNI database (adni. loni.usc.edu), an independent replication cohort. The ADNI was launched in 2003 as a public-private partnership under the leadership by Michael W. Weiner, MD, with a primary goal to test whether magnetic resonance imaging (MRI), positron emission tomography (PET), biological markers, as well as clinical and neuropsychological assessment can be combined to measure the progression of $\mathrm{MCI}$ and early $\mathrm{AD}$. For up-to-date information, see www.adni-info.org.

Our ADNI cohort included all the CN controls, MCI patients and AD patients with available baseline samples for CSF $\alpha$-synuclein. The inclusion/exclusion criteria are described at http://www.adni-info.org. In our study, we stratified the MCI group into stable MCI (sMCI) with no progression to $\mathrm{AD}$ dementia during at least 2-year follow-up, and progressive MCI (pMCI) with progression to $\mathrm{AD}$ dementia during at least 2-year follow-up. As a result, totally 4 groups were included: $\mathrm{CN}$ control, sMCI group, pMCI group and $\mathrm{AD}$ group. As to the 
"ATN" binary (i.e., positive or negative) categories, amyloid positive $(\mathrm{A}+)$ and negative $(\mathrm{A}-)$ were separated by a cutoff value of $192 \mathrm{pg} / \mathrm{ml}$ for CSF A $\beta$ level; Tau pathology positive $(\mathrm{T}+)$ and negative $(\mathrm{T}-)$ were separated by a cutoff value of $23 \mathrm{pg} / \mathrm{ml}$ for CSF phosphorylated tau (p-tau) level.

The CABLE study was approved by the Institutional Ethics Committee of Qingdao Municipal Hospital. Written informed consent was obtained from all study participants directly or from their guardians. The ADNI study was approved by the Institutional Review Board at each of the participating centers, and all participants provided written informed consent.

\section{CSF/plasma biomarker measurements}

CSF was collected by lumbar puncture through the L3/ L4 interspace and gently mixed to avoid gradient effects. The samples were then centrifuged at $2000 \mathrm{~g}$ for $10 \mathrm{~min}$ to remove cells and other insoluble materials, stored in 1 -ml aliquots at $-80^{\circ} \mathrm{C}$ until use for $\mathrm{A} \beta$ and tau analysis. CSF was sampled between 08:00 and 09:00 in the morning taking into account the possible circadian rhythm effect.

In the CABLE study, the concentrations of CSF A $\beta 42$, total tau (t-tau), $\mathrm{p}$-tau and CSF total $\alpha$-synuclein were measured separately using an enzyme-linked immunosorbent assay (ELISA) kit (LEGEND MAX ${ }^{\mathrm{mw}}$ Human $\alpha$ Synuclein ELISA Kit with pre-coated plate, Catalog No: 844101), according to the manufacturer's instructions. The samples and standards were measured in duplicate to generate an average value for the statistical analyses.

In the ADNI database, CSF $A \beta_{42}$, $\mathrm{t}$-tau and $\mathrm{p}$-tau were measured at the ADNI biomarker core (University of Pennsylvania) using the multiplex xMAP Luminex platform (Luminex Corp, Austin, TX, USA) with the INNOBIA AlzBio3 kit (Fujirebio, Ghent, Belgium). The CSF neurofilament light chain (NFL) concentrations were measured using a commercial ELISA kit (Uman Diagnostics). The plasma NFL concentrations were measured using an NFL kit (NF-light; Uman Diagnostics), transferred onto the ultrasensitive single-molecule array platform using a home brew kit (Simoa Homebrew Assay Development Kit; Quanterix Corporation). The levels of CSF total $\alpha$-synuclein concentrations in the ADNI cohort were measured by the Luminex MicroPlex Microspheres (Luminex Corp, Austin, TX), using the biotinylated goat anti-human $\alpha$-syn antibody (R\&D systems, Minneapolis, MN) as the detection antibody. The $\alpha$-synuclein Luminex assay demonstrated a low day-to-day and plate-to-plate signal variety. The accuracy of the assay was further determined by the recovery of spiked $\alpha$-synuclein protein, which was close to $93 \%$.

\section{Neuroimaging}

Structural MRI was performed only in the ADNI participants using a Siemens Trio 3.0 T scanner or Vision 1.5 T scanner (GE, Siemens and Philips). The regional volume estimates for the 1.5 and 3.0 T MRI images were processed with the Free-surfer software package version 4.3 and 5.1 image processing frameworks, respectively. The hippocampus and ventricles were selected as the regions of interest.

\section{Statistical analyses}

The associations between CSF $\alpha$-synuclein and demographic factors were analyzed with the Mann-Whitney test and the Spearman rank correlation test. The associations of CSF $\alpha$-synuclein with CSF A $\beta 42$, t-tau, and $p$ tau levels were analyzed with the linear regression after adjustment for age, gender, educational level, diagnosis and $A P O E \& 4$ genotype (with CSF $\alpha$-synuclein as a predictor). In the ADNI database, associations between CSF $\alpha$-synuclein concentrations and the diagnostic groups were tested in an analysis of covariance model adjusted for age, gender, educational level and $A P O E \varepsilon 4$ genotype. The effect of different CSF analytes on the risk of conversion to $A D$ was assessed with the logistic regression analysis. The receiver-operator curves and the area under the curves were derived from the predictive probabilities of the logistic regression models. We tested the associations of CSF $\alpha$-synuclein concentrations with longitudinal cognition and brain structure using the linear mixed-effects models. These models had random intercepts and slopes for time and an unstructured covariance matrix for the random effects and included the interaction between (continuous) time and CSF $\alpha$ synuclein as predictor with adjustment for confounders. All tests were 2-sided. Statistical significance was set at $P<0.05$. All regression analyses were corrected for age, gender, educational level, diagnosis, and $A P O E$ \&4 genotype. The following variables were natural logtransformed to ensure normality: CSF $\alpha$-synuclein, $\mathrm{p}$ tau, $\mathrm{t}$-tau, and $\mathrm{A} \beta$ levels, and hippocampus volume. All statistical analyses were performed using $R$ version 3.4.0 (R Foundation).

\section{Results}

\section{Characteristics of participants in the CABLE study}

We included 651 non-demented elders from the CABLE study, consisting of $457 \mathrm{CN}$ controls (238 females, $60.54 \pm 10.46$ years) and 194 MCI patients (109 females, $63.6 \pm 9.72$ years) (Table 1$)$. The $\mathrm{CN}$ individuals were significantly younger and more educated, and had significantly lower levels of CSF p-tau and t-tau, compared to the MCI participants. 
Table 1 Demographics of the study population in CABLE ${ }^{a}$

\begin{tabular}{|c|c|c|c|}
\hline & $\mathrm{CN}(n=457)$ & $\mathrm{MCl}(n=194)$ & $P$ value \\
\hline AGE, mean (SD), years & $60.93(10.55)$ & $65.44(10.01)$ & $<0.001$ \\
\hline Female, $n(\%)$ & $269(58.9)$ & $109(56.2)$ & 0.59 \\
\hline APOE $\varepsilon 4$ genotype carriers, $n(\%)$ & $69(15.1)$ & $35(18.0)$ & 0.41 \\
\hline Education, mean (SD), years & $10.38(6.12)$ & $8.56(4.23)$ & $<0.001$ \\
\hline CSF a-synuclein, mean (SD), ng/l & $1466.73(813.99)$ & $1501.19(914.13)$ & 0.61 \\
\hline CSF p-tau, mean (SD), ng/l & $38.11(9.69)$ & $40.04(12.42)$ & 0.03 \\
\hline CSF t-tau, mean (SD), ng/l & $173.3(77.96)$ & $191.02(122.57)$ & 0.03 \\
\hline $\mathrm{CSF} A \beta_{42}$, mean (SD), ng/l & $160.01(91.51)$ & $162.10(105.53)$ & 0.81 \\
\hline
\end{tabular}

$A \beta \beta$-amyloid, CN Cognitively normal, CSF Cerebrospinal fluid, MCI Mild cognitive impairment, MMSE Mini-Mental State Examination, $p$-tau Phosphorylated tau, $t$ tau Total tau.

${ }^{a} P$ values from the Kruskal-Wallis test or Fisher exact test

\section{CSF a-synuclein and established AD biomarkers in the CABLE study}

In the CABLE study, we examined the concentrations of CSF $\alpha$-synuclein and other established AD biomarkers (CSF A $\beta$, p-tau and $\mathrm{t}$-tau) and tested their relationships (Table 2). We found that the level of CSF $\alpha$-synuclein was positively associated with the CSF $\mathrm{t}$-tau $(\beta=0.56$, $P<0.001)$ and $\mathrm{p}$-tau $(\beta=0.35, P<0.001)$ among the nondemented participants. However, there was no association between CSF $\alpha$-synuclein and CSF A $\beta$ level at baseline. In addition, the same associations were found in the $\mathrm{CN}$ group and the MCI group (Table 2).

\section{Characteristics of participants in ADNI}

Three hundred and eighty-two subjects from the ADNI database were included (Table 3). This cohort consisted of $109 \mathrm{CN}$ controls (54 females, $75.63 \pm 5.22$ years), 117 sMCI patients ( 37 females, $74.34 \pm 7.60$ years), $66 \mathrm{pMCI}$ patients ( 25 females, $74.21 \pm 7.58$ years) and $90 \mathrm{AD}$ patients (39 females, $74.89 \pm 7.72$ years). According to the new "ATN" scheme, $258 \mathrm{~A}+(220 \mathrm{~A}+\mathrm{T}+)$ patients and 124 A- (96 A-T-) controls were included. As expected, the $\mathrm{AD}$ group had the highest frequency of the $A P O E \varepsilon 4$ allele $(69.23 \%)$ and the $\mathrm{CN}$ group had the lowest frequency $(23.85 \%)$. There was no significant difference in the educational level $(P=0.16)$ or age $(P=0.53)$ among these four groups. Furthermore, AD patients had lower
MMSE scores compared with the MCI patients and $\mathrm{CN}$ controls $(P<0.01)$.

\section{CSF a-synuclein and established AD biomarkers in ADNI} In the ADNI database, we found that the high CSF $\alpha$ synuclein levels were associated with the high CSF t-tau $(\beta=0.27, P<0.001)$ and $p$-tau $(\beta=0.36, P<0.001)$ in the whole cohort. However, there was no association between CSF $\alpha$-synuclein and CSF A $\beta$ level at baseline. The same results were obtained in the MCI group (CSF t-tau: $\beta=0.29, P<0.001$, CSF $p$-tau: $\beta=0.33, P<0.001$ ) and CN controls (CSF t-tau: $\beta=0.2, P<0.001$, CSF $p$ tau: $\beta=0.32, P<0.001)$. In addition, the CSF $\alpha$-synuclein concentration was associated with CSF NFL concentration in non-demented elders $(\beta=0.12, P<0.001)$. However, there was no association between CSF $\alpha$-synuclein and plasma NFL (Table 4, Fig. S1).

\section{CSF a-synuclein in different diagnostic groups in ADNI}

The level of CSF $\alpha$-synuclein showed a trend of increase with the progression of disease stage. The CSF $\alpha$ synuclein concentration was significantly higher in the $\mathrm{AD}$ and pMCI groups than in the $\mathrm{CN}$ controls $(P<$ 0.0001 and $P<0.001$, respectively) and the sMCI group ( $P=0.02$ and $P=0.04$, respectively) (Fig. 1a). In addition, the A+ AD group had higher CSF $\alpha$-synuclein levels than the A- controls $(P<0.001), \mathrm{A}+$ controls $(P<0.001)$, and A- MCI group $(P<0.001)$ (Fig. 1b). The A+ MCI

Table 2 Correlations between CSF a-synuclein and other biochemical markers in CABLE ${ }^{\text {a }}$

\begin{tabular}{|c|c|c|c|c|c|c|}
\hline & \multicolumn{2}{|c|}{ All participants } & \multicolumn{2}{|l|}{$\mathrm{CN}$} & \multicolumn{2}{|l|}{$\mathrm{MCl}$} \\
\hline & $\beta$ coefficient & $P$ value & $\beta$ coefficient & $P$ value & $\beta$ coefficient & $P$ value \\
\hline CSF t-tau & 0.56 & $<0.001$ & 0.38 & $<0.001$ & 0.67 & $<0.001$ \\
\hline CSF p-tau & 0.35 & $<0.001$ & 0.27 & $<0.001$ & 0.40 & $<0.001$ \\
\hline CSF A 442 & -0.02 & 0.97 & -0.01 & 0.82 & -0.07 & 0.69 \\
\hline
\end{tabular}

$A \beta \beta$-amyloid, CABLE Chinese Alzheimer's Biomarker and Lifestyle, CN Cognitively normal, CSF Cerebrospinal fluid, MCI Mild cognitive impairment, $p$-tau Phosphorylated tau, $t$-tau Total tau.

a Data are $\beta$ coefficients (with $P$ values) from linear regression models for correlations between CSF a-synuclein and other biomarkers, adjusted for age, gender, educational level and APOE \&4 genotype. Models were tested in the whole cohort and in individual diagnostic groups 
Table 3 Demographics of the study population in ADNI

\begin{tabular}{lllll}
\hline & CN $(\boldsymbol{n}=\mathbf{1 0 9})$ & $\mathbf{s M C l}(\boldsymbol{n}=\mathbf{1 1 7})$ & $\mathbf{p M C l}(\boldsymbol{n}=\mathbf{6 6})$ & AD $(\boldsymbol{n}=\mathbf{9 0})$ \\
\hline Age, mean (SD), years & $75.63(5.22)$ & $74.34(7.60)$ & $74.21(7.58)$ & $74.89(7.72)$ \\
Female, $n$ (\%) & $54(49.54)$ & $37(31.62)$ & $25(36.76)$ & $39(44.32)$ \\
APOE \&4 genotype carriers, $n$ (\%) & $26(23.85)$ & $55(47.00)$ & $42(61.76)$ & $63(69.23)$ \\
CSF a-synuclein, mean (SD), ng/L & $0.46(0.17)$ & $0.54(0.22)$ & $0.56(0.20)$ & $0.61(0.24)$ \\
MMSE score, mean (SD) & $29.07(1.05)$ & $27.15(1.64)$ & $26.58(1.77)$ & $23.39(1.80)$ \\
CSF Aß42, mean (SD), ng/L & $208.70(52.36)$ & $174.69(55.28)$ & $148.75(41.52)$ & $143.99(38.31)$ \\
CSF t-tau, mean (SD), ng/L & $69.08(29.85)$ & $97.31(64.77)$ & $112.00(41.52)$ & $122.83(57.09)$ \\
CSF p-tau, mean (SD), ng/L & $25.04(13.93)$ & $32.76(18.31)$ & $39.50(17.18)$ & $41.48(19.73)$ \\
Hippocampus volume, mm ${ }^{3}$ & $6648.16(766.59)$ & $5964.07(986.76)$ & $5522.46(1044.15)$ & $5217.39(1043.40)$ \\
\hline
\end{tabular}

Abbreviations: $A \beta \beta$-amyloid, $A D$ Alzheimer disease dementia, $C N$ Cognitively normal, CSF Cerebrospinal fluid, $s M C l$ Stable mild cognitive impairment, $p M C I$ Progressive mild cognitive impairment, MMSE Mini-Mental State Examination, $p$-tau Phosphorylated tau, $t$-tau Total tau

had higher CSF $\alpha$-synuclein levels than the A- controls $(P<0.01)$, A+ controls $(P<0.01)$, and the A- MCI group $(P=0.02)$. The CSF $\alpha$-synuclein level was also significantly different between the $\mathrm{A}+\mathrm{T}+$ group and the A-Tgroup $(P<0.0001)$ (Fig. 1c).

We generated receiver-operating curves based on the logistic regression models adjusted for age at baseline, gender, educational level and $A P O E \quad \varepsilon 4$ genotype to assess the predictive value of CSF $\alpha$ synuclein alone and in combination with other established AD biomarkers for the risk of conversion to AD. The area under the curve (AUC) of the baseline model containing CSF $\alpha$-synuclein, age at baseline, gender, educational level and $A P O E \& 4$ genotype was 0.76 in predicting the onset of $\mathrm{AD}$ among the $\mathrm{CN}$ controls, and the AUC was further increased by the inclusion of $C S F$ tau $/ A \beta$ ratio $(A U C=0.88$ ) (Fig. S2). As expected, the baseline model showed a similar predicting value for the onset of pMCI among the $\mathrm{CN}$ controls (Fig. S3). In the A-group, this baseline model showed a good predictive value for the risk of conversion to $\mathrm{A}+$ status $(\mathrm{AUC}=0.77$ ), and inclusion of CSF t-tau (AUC $=0.88$ ) and $\mathrm{p}$-tau (AUC $=0.92)$ further enhanced this predictive value (Fig. S4). Furthermore, the baseline model performed best when the participants were grouped by $A \beta$ deposition and pathology $(\mathrm{AUC}=0.84)$. We also detected that CSF $\alpha$-synuclein added value for diagnosis prediction (Fig. S5).

\section{CSF a-synuclein, longitudinal neuroimaging change and progression in ADNI}

Next, the linear mixed-effects models were utilized to test the associations between baseline CSF $\alpha$-synuclein concentration and subsequent disease progression, after adjustment for age, gender, educational level, diagnosis, and $A P O E \& 4$ genotype. The baseline CSF $\alpha$-synuclein concentration was found to be significantly associated with the hippocampal volume $(\beta=-0.008, P=0.001$ longitudinally) (Table 4, Fig. 2 (left)).

Table 4 Modelling the association of CSF biomarkers on AD biomarkers and clinical outcomes in ADNI ${ }^{\mathrm{a}}$

\begin{tabular}{|c|c|c|c|c|c|c|}
\hline \multirow[b]{2}{*}{ Cross-sectional (MR) } & \multicolumn{2}{|c|}{ All Participants } & \multicolumn{2}{|l|}{$\mathrm{MCl}$} & \multicolumn{2}{|l|}{$\mathrm{CN}$} \\
\hline & $\beta$ coefficient & $P$ value & $\beta$ coefficient & $P$ value & $\beta$ coefficient & $P$ value \\
\hline CSF t-tau & 0.27 & $<0.001$ & 0.29 & $<0.001$ & 0.20 & $<0.001$ \\
\hline CSF p-tau & 0.36 & $<0.001$ & 0.33 & $<0.001$ & 0.32 & $<0.001$ \\
\hline CSF A $A 42$ & -0.03 & 0.33 & -0.04 & 0.32 & 0.006 & 0.86 \\
\hline CSF NFL & 0.12 & $<0.001$ & 0.11 & 0.04 & 0.03 & 0.45 \\
\hline Plasma NFL & 0.04 & 0.27 & 0.02 & 0.73 & -0.04 & 0.53 \\
\hline \multicolumn{7}{|l|}{ Longitudinal (MELM) } \\
\hline Hippocampus & -0.008 & 0.001 & -0.007 & 0.04 & -0.003 & 0.17 \\
\hline Ventricles & 0.006 & 0.13 & 0.005 & 0.36 & 0.003 & 0.43 \\
\hline Cox (Hazard ratio) & Statistic & $P$ value & & & & \\
\hline MCI-to-AD dementia conversion & $1.53(1.15-2.0)$ & 0.004 & & & & \\
\hline
\end{tabular}

Abbreviations: CN Cognitively normal, CSF Cerebrospinal fluid, MCI Mild cognitive impairment, $p$-tau Phosphorylated tau, $t$-tau Total tau, Cox Cox proportional hazards model, MELM Mixed effects linear model, MR Multiple regression

${ }^{a}$ All models were adjusted for age, gender, educational level, APOE \&4 genotype and intracranial volume (for MRI only). Models were tested in the whole cohort and in individual diagnostic groups 


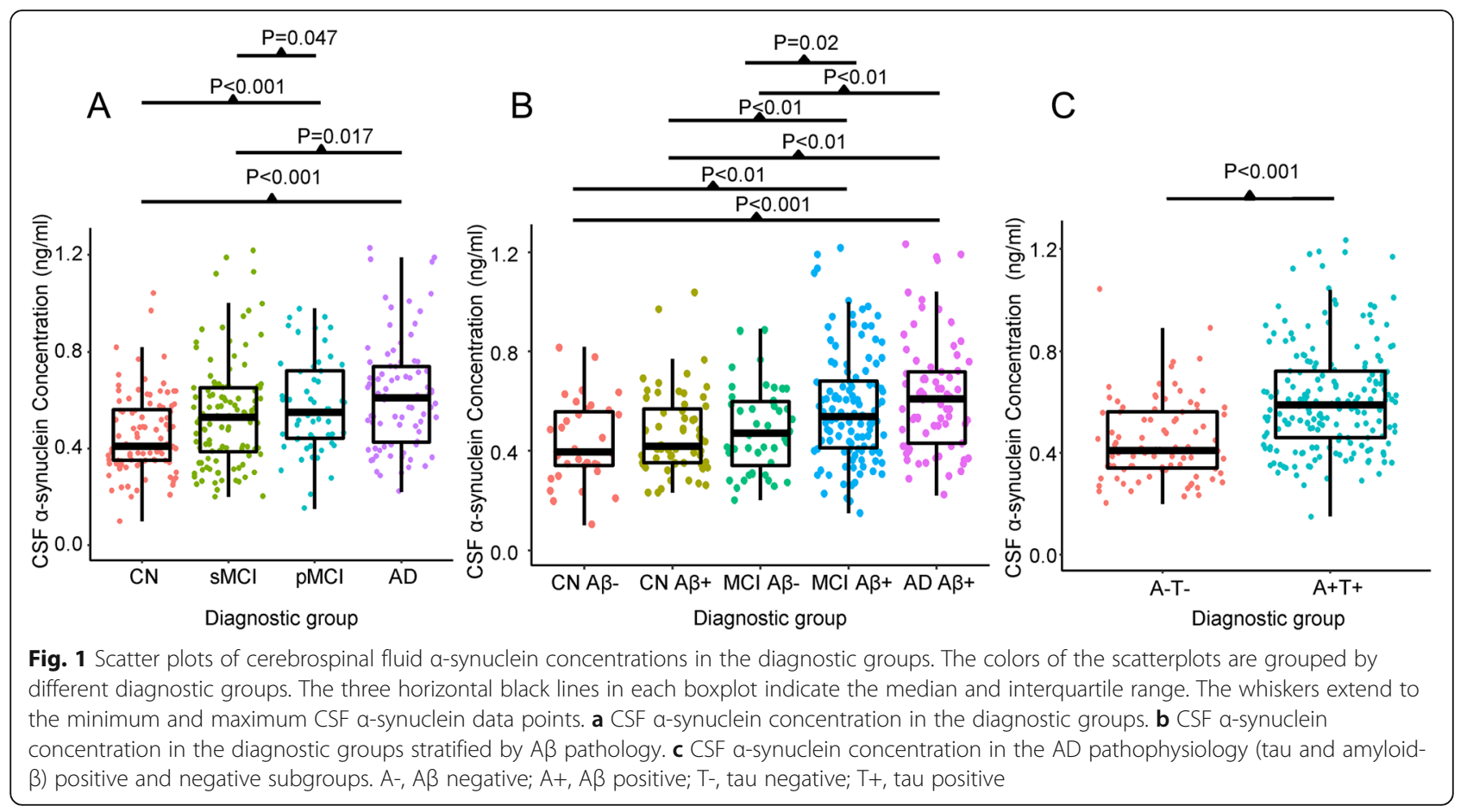

Figure 2 (right) presents the results of Kaplan-Meier analysis. The cox proportional hazards model was developed to estimate the predictive value of CSF $\alpha$ synuclein for the conversion risk from MCI to incidence of $\mathrm{AD}$ dementia, after controlling for baseline age, gender and years of education. MCI individuals with high CSF $\alpha$-synuclein levels would satisfy the diagnostic criteria for $\mathrm{AD}$ at a comparatively earlier interval (hazard ratio $2.79,95 \%$ CI $1.14-6.9, P=0.03$ ) (Table 4).

\section{Discussion}

In this study, we found that the CSF $\alpha$-synuclein concentration (1) was associated with CSF t-tau and p-tau levels among the non-demented elderly adults, (2) was elevated in the $\mathrm{AD}$ dementia group and the $\mathrm{A} \beta /$ tau-

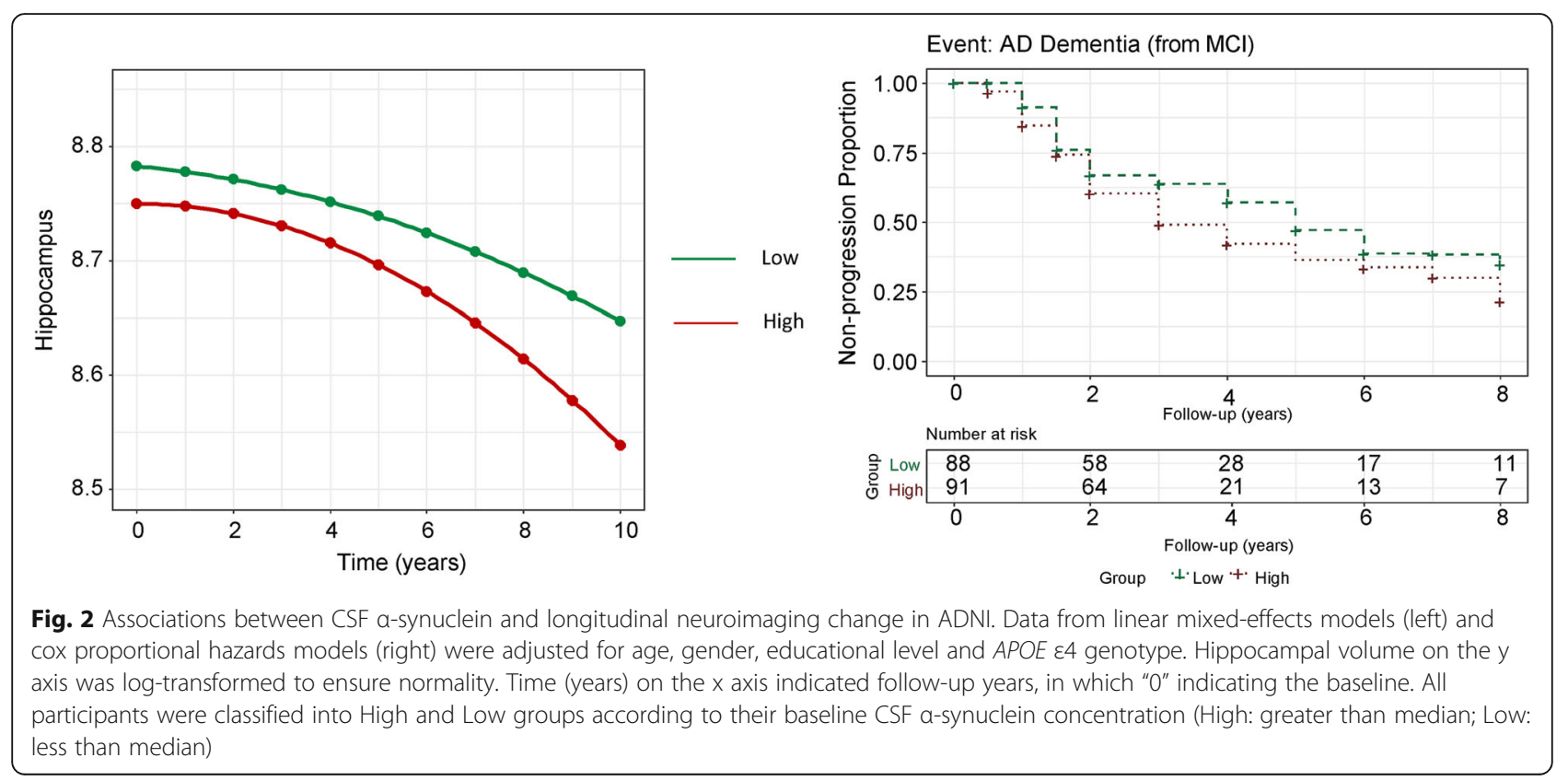


positive group compared with the control group, and (3) could predict hippocampal atrophy and the conversion from MCI to AD dementia. Taken together, these findings suggest that CSF $\alpha$-synuclein is a very early and potentially presymptomatic biomarker for $\mathrm{AD}$. This biomarker may be helpful for AD diagnosis and prediction of disease progression and staging of $\mathrm{AD}$ even in the preclinical stage.

"Pure" AD is characterized by the presence of both diffused neuritic plaques and intracellular neurofibrillary tangles, with a lack of abnormal $\alpha$-synuclein inclusions or neuritis. However, more than $50 \%$ of AD patients exhibit excessive brain accumulation of $\alpha$-synuclein-positive Lewy bodies, particularly in the amygdala [10,20]. The presence of $\alpha$-synuclein seems not to be innocuous, as these patients demonstrate an accelerated cognitive decline than subjects with AD alone [12, 21]. Previous studies have indicated that $\alpha$-synuclein can be secreted into the surrounding media in the brain and then to the CSF $[22,23]$. Therefore, the CSF could be used to investigate the mechanisms of $\alpha$-synuclein metabolism in the brain.

Consistent with most studies, our study showed that CSF $\alpha$-synuclein was higher in the AD group compared with the $\mathrm{CN}$ controls and $\mathrm{MCI}$ group. A possible hypothesis is that the higher level of $\alpha$-synuclein could induce a decrease in some proteins in synaptic vesicle and alterations of the protein composition of synaptic vesicles, thus causing neuronal damage in $\mathrm{AD}$, which in turn increases the release of $\alpha$-synuclein from damaged cells into the CSF $[24,25]$. As the CSF $\alpha$-synuclein levels are lower in synucleinopathies compared to control, but appear higher in $\mathrm{AD} / \mathrm{MCI}$ than control, the $\alpha$-synuclein may serve as a biomarker for differential dementia diagnosis. In this study, logistic regression analysis was used to assess the effect of CSF analytes on the risk of progression to $\mathrm{AD}$. The AUC (which reflects the predictive probabilities of the logistic regression models) of the model including CSF $\alpha$-synuclein, age at baseline, gender, educational level and $A P O E \& 4$ genotype had good performance in predicting progression from $\mathrm{CN}$ to pMCI or AD. Recently, the NIA-AA committee has recommended a different definition of $\mathrm{AD}$ by pathophysiology, independent of the clinical symptoms. They proposed that as long as biomarker evidence of $A \beta$ and tau pathology was present simultaneously, the term "Alzheimer's disease" would be applied. Here the CSF $\alpha$ synuclein model had high diagnostic accuracy for patients with the diagnosis of AD based on the "ATN" system $(\mathrm{A}+\mathrm{T}+)$ vs controls $(\mathrm{A}-\mathrm{T}-)(\mathrm{AUC}=0.84$, which was comparable to other established CSF biomarkers).

Many lines of evidence have suggested that the pathological $\alpha$-synuclein, $A \beta$ and tau have synergistic adverse effects to promote the aggregation of each other, thereby amplifying the neuronal damage [24, 26-31]. Notably, $\alpha-$ synuclein inclusions are commonly observed in patients with familial Down's syndrome, where $A \beta$ peptides are highly expressed. In both diseases, $\alpha$-synuclein affects the biological pathways and promotes the formation of A $\beta$ aggregates. $\alpha$-Synuclein has also been proposed to be implicated in synaptic vesicle formation, axonal transport as well as dopamine synthesis and metabolism [32]. In normal conditions, the synaptic membrane is integrated and the $\alpha$-synuclein is completely released into the cytosol. However, in the event of neuronal damage and synaptic membrane defect, both aggregated $A \beta$ and $\alpha$-synuclein might attach to synaptic membrane and accumulate in lipid rafts. The synaptic membrane-bound $\alpha$-synuclein could not only induce cytosolic $\alpha$-synuclein to aggregate as intracellular Lewy bodies but also interact with the membrane-associated $A \beta_{40}$ and $A \beta_{42}$ peptides [33]. This may explain the low level of CSF $\alpha$ synuclein in individuals with normal cognitive function to a certain extent. Moreover, an in vitro experiment has demonstrated that the interaction with $A \beta_{1-42}$ is sufficient to induce the intracellular accumulation of $\alpha$ synuclein, whereas interaction with $A \beta_{1-40}$ is not [34]. In our study, however, we did not find any association between CSF $\alpha$-synuclein and CSF A $\beta$ levels at baseline. The reason may be that this mutual effect occurs in the initial stages of the mixed pathology, preceding the presence of intracellular $\alpha$-synuclein in surrounding media and eventually in the CSF by years or decades. We only studied the CSF total $\alpha$-synuclein level rather than the oligomeric or phosphorylated forms. Future studies focusing on the oligomeric or phosphorylated forms of $\alpha$ synuclein may provide additional information.

Moreover, $\alpha$-synuclein has also being observed in progressive supranuclear palsy [35] and frontotemporal dementia [36]. Many studies have proposed that $\alpha$ synuclein and tau interact to promote the fibrillation and toxicity of each other [26]. However, unlike $\alpha$ synuclein that could spontaneously polymerize into amyloidogenic fibrils, tau requires cofactors such as glycosaminoglycans or nucleic acids to polymerize [37]. The $\alpha$ synuclein polymers act as amyloidogenic "seeds" or as amyloidogenic chaperones that induce the formation of tau fibrillary inclusions even in the absence of $\alpha$ synuclein coexpression $[26,27,38]$. Besides, Tau promotes $\alpha$-synuclein to polymerize into fibrils. Low concentrations of $\alpha$-synuclein do not fibrillize without tau, however, in the presence of tau, most $\alpha$-synuclein assembles into fibrils. Much attention has been paid to the relationship between CSF $\alpha$-synuclein and tau. Consistent with most studies [24, 29], our study found positive associations of CSF $\alpha$-synuclein with CSF $t$-tau and $p$ tau levels in the CABLE study. We noted that the mean values for CSF $\alpha$-synuclein and CSF A $\beta$ levels between controls in the 2 Chinese cohorts using similar assays 
were different. This could partly be explained by the differences in pre-analytical protocols, analytical procedures, assay quality and the absolute levels between assay formats [39]. In addition, the CSF $\alpha$-synuclein also correlated with the CSF NFL in the whole cohort, but not in the $\mathrm{CN}$ group, suggesting that they were confounded by diagnosis. This finding probably reflects that several different pathological conditions (e.g., degeneration of different types of axons) may drive the different biomarker responses. We also tested the association between CSF $\alpha$-synuclein and plasma NFL concentration, but did not find any significant association. More studies with larger sample sizes are needed to clarify whether $\alpha$ synuclein and NFL reflect the same neurodegeneration pattern.

Importantly, we found that the CSF $\alpha$-synuclein levels might correlate with $\mathrm{AD}$ severity and progression, which was consistent with a recent study indicating that increased $\alpha$-synuclein displayed a stronger association with cognitive impairment than soluble $\mathrm{A} \beta$ and tau levels [40]. It has been widely recognized that $\alpha$-synuclein is a synaptic marker. $\alpha$-Synuclein is highly expressed in the pre-synaptic terminals $[41,42]$ and plays a role in the regulation of neurotransmitter release, synaptic function and plasticity. It could trigger synaptoxicity not only by directly damaging the synaptic membrane, but also by damaging the mitochondria, lysosomes, or microtubules, leading to dendritic and spine alterations, axonal dystrophy, and eventually neuronal loss [43]. Along with the synaptic damage, $\alpha$-synuclein is released into the CSF. Therefore, it is reasonable to assume that the CSF $\alpha$ synuclein level correlates with cognitive decline in $\mathrm{AD}$, since synaptic damage is supposed to be a strong predictor of cognitive decline [44].

\section{Conclusions}

CSF $\alpha$-synuclein was associated with CSF t-tau and $\mathrm{p}$ tau levels among the non-demented elderly adults. In the ADNI database, CSF $\alpha$-synuclein concentrations were increased with the severity of the disease. CSF $\alpha$-synuclein predicted longitudinal hippocampus atrophy and conversion from MCI to AD dementia. The current findings suggest CSF $\alpha$-synuclein as a very early and potentially presymptomatic biomarker for $\mathrm{AD}$, a prognostic marker in the clinic, and an outcome measure in clinical trials.

\section{Supplementary Information}

The online version contains supplementary material available at https://doi. org/10.1186/s40035-020-00222-1.

Additional file 1: Supplementary Figure S1. Association between CSF a-synuclein and CSF/plasma NFL concentration. Linear regression trend lines are shown in blue. These regression lines were unadjusted, while the corresponding analysis in the text was adjusted for age, sex, educational level, and APOE \&4 genotype. CSF/plasma NFL and CSF asynuclein concentrations on the $y$ - and $x$-axes were logarithmic.

Additional file 2: Supplementary Figure S2. Receiver operating curves to assess the diagnostic accuracy of CSF a-synuclein and other biomarkers for AD dementia. Receiver operating curves of logistic regression model were controlled for age at baseline, gender, educational level and $A P O E$ \&4 genotype.

Additional file 3: Supplementary Figure S3. Conversion from CN to $\mathrm{pMCl}$ as predicted by baseline CSF biomarkers. Receiver operating curves of the logistic regression model controlling for age at baseline, gender, educational level and APOE \&4 genotype for predicting conversion to $\mathrm{pMCl}$ among people with normal cognitive function.

Additional file 4: Supplementary Figure S4. Conversion from ABnegative status to $A \beta$-positive group as predicted by the baseline CSF biomarkers. Receiver operating curves of the logistic regression model were controlled for age at baseline, gender, educational level and APOE $\varepsilon 4$ genotype for predicting conversion to $A \beta$-positive status among the A $\beta$-negative group.

Additional file 5: Supplementary Figure S5. Receiver operating curves for predicting conversion from AD pathophysiology (tau and amyloid- $\beta$ ) negative to positive. The baseline model included age at baseline, gender, educational level and APOE $\varepsilon 4$ genotype.

\section{Abbreviations}

ADNI: Alzheimer's Disease Neuroimaging Initiative; $A \beta$ : $\beta$-amyloid: AD: Alzheimer's disease; CSF: Cerebrospinal fluid; CABLE: Chinese Alzheimer's Biomarker and Life style; CN: Cognitively normal; CDR: Clinical Dementia Rating; DLB: Dementia with Lewy bodies; MMSE: Mini-Mental State Examination; MRI: Magnetic resonance imaging; MCl: Mild cognitive impairment; PET: Positron emission tomography; PD: Parkinson's disease

\section{Acknowledgements}

Data used in preparation of this paper were obtained from the Alzheimer's Disease Neuroimaging Initiative (ADNI) database (http://adni.loni.usc.edu/). As such, the investigators within the ADNI contributed to the design and implementation of ADNI and/or provided data but did not participate in the analysis or in the writing of this paper. A complete listing of ADNI investigators can be found at http://adni.loni.usc.edu/wp-content/uploads/ how_to_apply/ADNI_Acknowledgement_List.pdf.

\section{Authors' contributions}

Dr. Jin-Tai Yu had full access to all the data in the study and was responsible for the integrity of the data and the accuracy of the data analysis. Study concept and design: Jin-Tai Yu, Jie-Qiong Li. Acquisition, analysis, or interpretation of data: All authors. Drafting of the manuscript: Jie-Qiong Li. Critical revision of the manuscript for important intellectual content: Jin-Tai Yu, John Suckling, Hui-Fu Wang, Qiang Dong, Lan Tan, Qiang Liu, Yan-Jiang Wang, Chuan-Tao Zuo, Can Zhang. Statistical analysis: Jie-Qiong Li. Obtained funding: Jin-Tai Yu. The author(s) read and approved the final manuscript.

\section{Funding}

This study was supported by grants from the National Natural Science Foundation of China (91849126), the National Key R\&D Program of China (2018YFC1314700), Shanghai Municipal Science and Technology Major Project (No.2018SHZDZX01), ZJLab, Shanghai Center for Brain Science and Brain-Inspired Technology, Tianqiao and Chrissy Chen Institute, and the State Key Laboratory of Neurobiology and Frontiers Center for Brain Science of Ministry of Education, Fudan University. Data collection and sharing for this project was funded by the Alzheimer's Disease Neuroimaging Initiative (ADNI) (National Institutes of Health Grant U01 AG024904) and DOD ADNI (Department of Defense award number W81XWH-12-2-0012). ADNI is funded by the National Institute on Aging, the National Institute of Biomedical Imaging and Bioengineering, and through generous contributions from the following: AbbVie, Alzheimer's Association; Alzheimer's Drug Discovery Foundation; Araclon Biotech; BioClinica, Inc.;Biogen; Bristol-Myers Squibb Company; CereSpir, Inc.; Cogstate; Eisai Inc.; Elan Pharmaceuticals, Inc.; Eli Lilly and Company; Eurolmmun; F. Hoffmann-La Roche Ltd. and its affiliated 
company Genentech, Inc.; Fujirebio; GE Healthcare; IXICO Ltd.; Janssen Alzheimer Immunotherapy Research \& Development, LLC.; Johnson \& Johnson Pharmaceutical Research \& Development LLC.; Lumosity; Lundbeck; Merck \& Co., Inc.; Meso Scale Diagnostics, LLC.; NeuroRx Research; Neurotrack Technologies; Novartis Pharmaceuticals Corporation; Pfizer Inc.; Piramal Imaging; Servier; Takeda Pharmaceutical Company; and Transition Therapeutics. The Canadian Institutes of Health Research is providing funds to support ADNI clinical sites in Canada. Private sector contributions are facilitated by the Foundation for the National Institutes of Health (www.fnih.org). The grantee organization is the Northern California Institute for Research and Education, and the study is coordinated by the Alzheimer's Therapeutic Research Institute at the University of Southern California. ADNI data are disseminated by the Laboratory for Neuro Imaging at the University of Southern California.

\section{Availability of data and materials}

Not applicable.

\section{Ethics approval and consent to participate}

The CABLE study was approved by the Institutional Ethics Committee of Qingdao Municipal Hospital. The ADNI study was approved by the institutional review boards of all participating centres (https://adni.loni.usc. edu/wp-content/uploads/how_to_apply/ADNI_Acknowledgement_List.pdf), including: Oregon Health and Science University; University of Southern California; University of California—San Diego; University of Michigan; Mayo Clinic, Rochester; Baylor College of Medicine; Columbia University Medical Center; Washington University, St. Louis; University of Alabama at Birmingham; Mount Sinai School of Medicine; Rush University Medical Center; Wien Center; Johns Hopkins University; New York University; Duke University Medical Center; University of Pennsylvania; University of Kentucky; University of Pittsburgh; University of Rochester Medical Center; University of California, Irvine; University of Texas Southwestern Medical School; Emory University; University of Kansas, Medical Center; University of California, Los Angeles; Mayo Clinic, Jacksonville; Indiana University; Yale University School of Medicine; McGill University, Montreal-Jewish General Hospital; Sunnybrook Health Sciences, Ontario; U.B.C. Clinic for AD \& Related Disorders; Cognitive Neurology — St. Joseph's, Ontario; Cleveland Clinic Lou Ruvo Center for Brain Health; Northwestern University; Premiere Research Inst (Palm Beach Neurology); Georgetown University Medical Center; Brigham and Women's Hospital; Stanford University; Banner Sun Health Research Institute; Boston University; Howard University; Case Western Reserve University; University of California, Davis_Sacramento; Neurological Care of CNY; Parkwood Hospital; University of Wisconsin; University of California, Irvine—BIC; Banner Alzheimer's Institute; Dent Neurologic Institute; Ohio State University; Albany Medical College; Hartford Hospital, Olin Neuropsychiatry Research Center; Dartmouth-Hitchcock Medical Center; Wake Forest University Health Sciences; Rhode Island Hospital; Butler Hospital; UC San Francisco; Medical University South Carolina; St. Joseph's Health Care Nathan Kline Institute; University of lowa College of Medicine; Cornell University and University of South Florida: USF Health Byrd Alzheimer's Institute. Written informed consent was obtained from all participants or their authorized representatives The investigators within the ADNI contributed to the design and implementation of the ADNI and/or provided data but did not participate in analysis or writing of this report. A complete listing of ADNI investigators can be found online (http://adni.loni.usc.edu/wp-content/uploads/how_to_apply/ ADNI_Acknowledgement_List.pdf).

\section{Consent for publication}

Not applicable.

\section{Competing interests}

The authors declare that they have no competing interests.

\section{Author details}

'Department of Neurology and Institute of Neurology, Huashan Hospital, Shanghai Medical College, Fudan University, Shanghai 20040, China. ${ }^{2}$ Department of Neurology, Qingdao Municipal Hospital, Qingdao University, Qingdao 266071, China. ${ }^{3}$ Department of Neurology, The Affiliated Hospital of Qingdao University, Qingdao 266071, China. ${ }^{4}$ Department of Anesthesiology, Qingdao Municipal Hospital, Qingdao University, Qingdao 266071, China. ${ }^{5}$ Department of Neurology and Center for Clinical Neuroscience, Daping Hospital, Third Military Medical University, Chongqing 400042, China.
Received: 24 May 2020 Accepted: 5 November 2020

Published online: 23 November 2020

\section{References}

1. Eichler T, Thyrian JR, Hertel J, Kohler L, Wucherer D, Dreier A, et al. Rates of formal diagnosis in people screened positive for dementia in primary care: results of the DelpHi-trial. J Alzheimer's Dis. 2014;42(2):451-8.

2. Sperling RA, Aisen PS, Beckett LA, Bennett DA, Craft S, Fagan AM, et al. Toward defining the preclinical stages of Alzheimer's disease: recommendations from the National Institute on Aging-Alzheimer's Association workgroups on diagnostic guidelines for Alzheimer's disease. Alzheimers Dement. 2011;7(3):280-92.

3. Jessen F, Amariglio RE, van Boxtel M, Breteler M, Ceccaldi M, Chetelat G, et al. A conceptual framework for research on subjective cognitive decline in preclinical Alzheimer's disease. Alzheimers Dement. 2014;10(6):844-52.

4. Jack CR Jr, Bennett DA, Blennow K, Carrillo MC, Feldman HH, Frisoni GB, et al. A/T/N: an unbiased descriptive classification scheme for Alzheimer disease biomarkers. Neurology. 2016;87(5):539-47.

5. Sonnen JA, Larson EB, Crane PK, Haneuse S, Li G, Schellenberg GD, et al. Pathological correlates of dementia in a longitudinal, population-based sample of aging. Ann Neurol. 2007;62(4):406-13.

6. James BD, Wilson RS, Boyle PA, Trojanowski JQ, Bennett DA, Schneider JA. TDP-43 stage, mixed pathologies, and clinical Alzheimer's-type dementia. Brain. 2016;139:2983-93.

7. Jack CR Jr, Bennett DA, Blennow K, Carrillo MC, Dunn B, Haeberlein SB, et al. NIA-AA research framework: toward a biological definition of Alzheimer's disease. Alzheimers Dement. 2018;14(4):535-62.

8. Echavarri C, Burgmans S, Caballero MC, Garcia-Bragado F, Verhey FR, Uylings HB. Co-occurrence of different pathologies in dementia: implications for dementia diagnosis. J Alzheimer's Dis. 2012;30(4):909-17.

9. Kovacs GG. Clinical stratification of subtypes of Alzheimer's disease. Lancet Neurol. 2012;11(10):839-41.

10. Hamilton RL. Lewy bodies in Alzheimer's disease: a neuropathological review of 145 cases using alpha-synuclein immunohistochemistry. Brain Pathol. 2000;10(3):378-84.

11. Kraybill ML, Larson EB, Tsuang DW, Teri L, McCormick WC, Bowen JD, et al. Cognitive differences in dementia patients with autopsy-verified AD, Lewy body pathology, or both. Neurology. 2005;64(12):2069-73.

12. Olichney JM, Galasko D, Salmon DP, Hofstetter CR, Hansen LA, Katzman R, et al. Cognitive decline is faster in Lewy body variant than in Alzheimer's disease. Neurology. 1998;51(2):351-7.

13. El-Agnaf OM, Salem SA, Paleologou KE, Cooper LJ, Fullwood NJ, Gibson MJ, et al. Alpha-synuclein implicated in Parkinson's disease is present in extracellular biological fluids, including human plasma. FASEB J. 2003;17(13): 1945-7.

14. Mollenhauer B, Cullen V, Kahn I, Krastins B, Outeiro TF, Pepivani I, et al. Direct quantification of CSF alpha-synuclein by ELISA and first crosssectional study in patients with neurodegeneration. Exp Neurol. 2008;213(2): $315-25$.

15. Hansson O, Hall S, Ohrfelt A, Zetterberg H, Blennow K, Minthon L, et al. Levels of cerebrospinal fluid alpha-synuclein oligomers are increased in Parkinson's disease with dementia and dementia with Lewy bodies compared to Alzheimer's disease. Alzheimer Res Ther. 2014;6(3):25.

16. Lim X, Yeo JM, Green A, Pal S. The diagnostic utility of cerebrospinal fluid alpha-synuclein analysis in dementia with Lewy bodies - a systematic review and meta-analysis. Parkinsonism Relat Disord. 2013;19(10):851-8.

17. Parnetti L, Chiasserini D, Persichetti E, Eusebi P, Varghese S, Qureshi MM et al. Cerebrospinal fluid lysosomal enzymes and alpha-synuclein in Parkinson's disease. Mov Disord. 2014;29(8):1019-27.

18. Mollenhauer B, Locascio JJ, Schulz-Schaeffer W, Sixel-Doring F, Trenkwalder C, Schlossmacher MG. Alpha-Synuclein and tau concentrations in cerebrospinal fluid of patients presenting with parkinsonism: a cohort study. Lancet Neurol. 2011;10(3):230-40.

19. Chiasserini D, Biscetti L, Eusebi P, Salvadori N, Frattini G, Simoni S, et al. Differential role of CSF fatty acid binding protein 3, alpha-synuclein, and Alzheimer's disease core biomarkers in Lewy body disorders and Alzheimer's dementia. Alzheimer Res Ther. 2017;9(1):52.

20. Irizarry MC, Kim TW, McNamara M, Tanzi RE, George JM, Clayton DF, et al. Characterization of the precursor protein of the non-a beta component of senile plaques (NACP) in the human central nervous system. J Neuropathol Exp Neurol. 1996;55(8):889-95. 
21. Parkkinen $L$, Soininen $H$, Alafuzoff I. Regional distribution of alpha-synuclein pathology in unimpaired aging and Alzheimer disease. J Neuropathol Exp Neurol. 2003;62(4):363-7.

22. Borghi R, Marchese R, Negro A, Marinelli L, Forloni G, Zaccheo D, et al. Full length alpha-synuclein is present in cerebrospinal fluid from Parkinson's disease and normal subjects. Neurosci Lett. 2000;287(1):65-7.

23. Tokuda T, Salem SA, Allsop D, Mizuno T, Nakagawa M, Qureshi MM, et al. Decreased alpha-synuclein in cerebrospinal fluid of aged individuals and subjects with Parkinson's disease. Biochem Biophys Res Commun. 2006; 349(1):162-6.

24. Toledo JB, Korff A, Shaw LM, Trojanowski JQ, Zhang J. CSF alpha-synuclein improves diagnostic and prognostic performance of CSF tau and Abeta in Alzheimer's disease. Acta Neuropathol. 2013;126(5):683-97.

25. Nemani VM, Lu W, Berge V, Nakamura K, Onoa B, Lee MK, et al. Increased expression of alpha-synuclein reduces neurotransmitter release by inhibiting synaptic vesicle reclustering after endocytosis. Neuron. 2010;65(1):66-79.

26. Giasson BI, Forman MS, Higuchi M, Golbe LI, Graves CL, Kotzbauer PT, et al. Initiation and synergistic fibrillization of tau and alpha-synuclein. Science. 2003;300(5619):636-40

27. Lee VM, Giasson BI, Trojanowski JQ. More than just two peas in a pod: common amyloidogenic properties of tau and alpha-synuclein in neurodegenerative diseases. Trends Neurosci. 2004;27(3):129-34.

28. Clinton LK, Blurton-Jones M, Myczek K, Trojanowski JQ, LaFerla FM. Synergistic interactions between Abeta, tau, and alpha-synuclein: acceleration of neuropathology and cognitive decline. J Neurosci. 2010; 30(21):7281-9.

29. Slaets S, Vanmechelen E, Le Bastard N, Decraemer H, Vandijck M, Martin JJ, et al. Increased CSF alpha-synuclein levels in Alzheimer's disease: correlation with tau levels. Alzheimers Dement. 2014;10(5 Suppl):S290-8.

30. Resende R, Marques SC, Ferreiro E, Simoes I, Oliveira CR, Pereira CM. Effect of alpha-synuclein on amyloid beta-induced toxicity: relevance to Lewy body variant of Alzheimer disease. Neurochem Res. 2013;38(4):797-806.

31. Zhang J, Mattison HA, Liu C, Ginghina C, Auinger P, McDermott MP, et al. Longitudinal assessment of tau and amyloid beta in cerebrospinal fluid of Parkinson disease. Acta Neuropathol. 2013;126(5):671-82.

32. Sidhu A, Wersinger $C$, Vernier $P$. Alpha-Synuclein regulation of the dopaminergic transporter: a possible role in the pathogenesis of Parkinson's disease. FEBS Lett. 2004;565(1-3):1-5.

33. Mandal PK, Pettegrew JW, Masliah E, Hamilton RL, Mandal R. Interaction between Abeta peptide and alpha synuclein: molecular mechanisms in overlapping pathology of Alzheimer's and Parkinson's in dementia with Lewy body disease. Neurochem Res. 2006;31(9):1153-62.

34. Masliah E, Rockenstein E, Veinbergs I, Sagara Y, Mallory M, Hashimoto M, et al. Beta-amyloid peptides enhance alpha-synuclein accumulation and neuronal deficits in a transgenic mouse model linking Alzheimer's disease and Parkinson's disease. Proc Natl Acad Sci U S A. 2001;98(21):12245-50.

35. Judkins AR, Forman MS, Uryu K, Hinkle DA, Asbury AK, Lee VM, et al. Cooccurrence of Parkinson's disease with progressive supranuclear palsy. Acta Neuropathol. 2002;103(5):526-30.

36. Yancopoulou D, Xuereb JH, Crowther RA, Hodges JR, Spillantini MG. Tau and alpha-synuclein inclusions in a case of familial frontotemporal dementia and progressive aphasia. J Neuropathol Exp Neurol. 2005;64(3):245-53.

37. Paudel HK, Li W. Heparin-induced conformational change in microtubuleassociated protein tau as detected by chemical cross-linking and phosphopeptide mapping. J Biol Chem. 1999;274(12):8029-38.

38. Waxman EA, Giasson BI. Induction of intracellular tau aggregation is promoted by alpha-synuclein seeds and provides novel insights into the hyperphosphorylation of tau. J Neurosci. 2011;31(21):7604-18.

39. Dubois B, Hampel H, Feldman HH, Scheltens P, Aisen P, Andrieu S, et al. Preclinical Alzheimer's disease: definition, natural history, and diagnostic criteria. Alzheimers Dement. 2016;12(3):292-323.

40. Larson ME, Sherman MA, Greimel S, Kuskowski M, Schneider JA, Bennett DA, et al. Soluble alpha-synuclein is a novel modulator of Alzheimer's disease pathophysiology. J Neurosci. 2012;32(30):10253-66.

41. Iwai A, Masliah E, Yoshimoto M, Ge N, Flanagan L, de Silva HA, et al. The precursor protein of non-a beta component of Alzheimer's disease amyloid is a presynaptic protein of the central nervous system. Neuron. 1995;14(2): 467-75

42. Jakes R, Spillantini MG, Goedert M. Identification of two distinct synucleins from human brain. FEBS Lett. 1994:345(1):27-32.
43. Kester MI, Teunissen CE, Crimmins DL, Herries EM, Ladenson JH, Scheltens $P$, et al. Neurogranin as a cerebrospinal fluid biomarker for synaptic loss in symptomatic Alzheimer disease. JAMA Neurol. 2015;72(11):1275-80.

44. Masliah E, Mallory M, Hansen L, DeTeresa R, Alford M, Terry R. Synaptic and neuritic alterations during the progression of Alzheimer's disease. Neurosci Lett. 1994;174(1):67-72.

\section{Ready to submit your research? Choose BMC and benefit from:}

- fast, convenient online submission

- thorough peer review by experienced researchers in your field

- rapid publication on acceptance

- support for research data, including large and complex data types

- gold Open Access which fosters wider collaboration and increased citations

- maximum visibility for your research: over $100 \mathrm{M}$ website views per year

At BMC, research is always in progress.

Learn more biomedcentral.com/submissions 